\title{
SASAKIAN MANIFOLD WITH PSEUDO-RIEMANNIAN METRIC
}

\author{
TOSHIO TAKAHASHI *) \\ (Received December 4, 1968)
}

Introduction. Sasakian manifold with Riemannian metric is defined by S. Sasaki [5]. In this paper, we want to define Sasakian manifold with pseudo-Riemannian metric, and discuss the classification of Sasakian manifolds.

In section 1, we define a Sasakian manifold (with pseudo-Riemannian metric). In section 2, we define the model spaces of Sasakian manifolds which are used in section 4 for the classification of Sasakian manifolds of constant $\phi$-sectional curvatures. In section 3 , we discuss $D$-homothetic deformation which is defined by S. Tanno [9], and prove some fundamental lemmas concerning completeness of the deformed metric. In section 5, we prove that a Sasakian manifold, satisfying $R(X, Y) \cdot R=0$ for all tangent vectors $X$ and $Y$, is of constant curvature. In section 6 , we discuss a Sasakian manifold $M^{2 n+1}$ which is properly and isometrically immersed in $E_{s}^{2 n+2}$.

I wish to express my hearty thanks to Prof. K. Nomizu, Prof. S. Sasaki and Prof. S. Tanno for their valuable advices.

1. Preliminaries. Manifolds and tensor fields are supposed to be of class $C^{\infty}$.

Let $M=M^{2 n+1}$ be a connected differentiable manifold, and let $\phi, \xi$ and $\eta$ be tensor fields of type $(1,1),(1,0)$ and $(0,1)$, respectively, on $M$.

DEFinition. $(\phi, \xi, \eta)$ is called an almost contact structure on $M$, if the followings are satisfied:

$$
\begin{aligned}
& \eta(\xi)=1, \\
& \eta(\phi(X))=0, \quad X \in \mathfrak{X}(M), \\
& \phi^{2}(X)=-X+\eta(X) \xi, \quad X \in \mathfrak{X}(M) .
\end{aligned}
$$

*) Partially supported by the National Science Foundation. 
DEFINITION. $(\phi, \xi, \eta, g, \varepsilon)$ is called an almost contact metric structure on $M$, if $(\phi, \xi, \eta)$ is an almost contact structure on $M$ and $g$ is a pseudoRiemannian metric on $M$ such that

$$
\begin{aligned}
& g(\xi, \xi)=\varepsilon, \quad \varepsilon=+1 \text { or }-1, \\
& \eta(X)=\varepsilon g(\xi, X), \quad X \in \mathfrak{X}(M), \\
& g(\phi X, \phi Y)=g(X, Y)-\varepsilon \eta(X) \eta(Y), \quad X, Y \in \mathfrak{X}(M) .
\end{aligned}
$$

DEFINITION. $(\phi, \xi, \eta, g, \varepsilon)$ is a contact metric structure on $M$, if it is an almost contact metric structure on $M$ and satisfies

$$
d \eta(X, Y)=g(\phi X, Y), \quad X, Y \in \mathfrak{X}(M) .
$$

DEFINITION. $(\phi, \xi, \eta, g, \varepsilon)$ is a normal contact metric structure on $M$, if it is a contact metric structure and satisfies

$$
\left(\nabla_{x} \phi\right) Y=\varepsilon \eta(Y) X-g(X, Y) \xi, \quad X, Y \in \mathfrak{X}(M),
$$

where $\nabla$ indicates the Levi-Civita connection for the pseudo-Riemannian metric $g$. In this case, we call $M(\phi, \xi, \eta, g, \varepsilon)$ a Sasakian manifold.

The following example shows that we may assume $\varepsilon=1$ without loss of generality.

EXAMPLE. Let $(\phi, \xi, \eta, g, \varepsilon)$ be an almost contact metric structure (resp. a normal contact metric structure) on $M$. We put

$$
\bar{g}=-g, \quad \bar{\xi}=-\xi, \quad \bar{\eta}=-\eta, \quad \bar{\phi}=\phi .
$$

Then $(\bar{\phi}, \bar{\xi}, \bar{\eta}, \bar{g}, \bar{\varepsilon}), \bar{\varepsilon}=-\varepsilon$, is an almost contact metric structure (resp. a normal contact metric structure) on $M$.

Proof. It is easy to see that $(\bar{\phi}, \bar{\xi}, \bar{\eta}, \bar{g}, \bar{\varepsilon})$ is an almost contact metric structure, and it is a contact metric structure if $(\phi, \xi, \eta, g, \varepsilon)$ is a contact metric structure. Suppose $(\phi, \xi, \eta, g, \varepsilon)$ is a normal contact metric structure. Since the parallelism with respect to $g$ and the parallelism with respect to $\bar{g}$ are the same, we get

$$
\begin{aligned}
\left(\bar{\nabla}_{x} \bar{\phi}\right) Y & =\left(\nabla_{x} \phi\right) Y \\
& =\varepsilon \eta(Y) X-g(X, Y) \xi \\
& =\bar{\varepsilon} \bar{\eta}(Y) X-\bar{g}(X, Y) \bar{\xi}
\end{aligned}
$$


Thus $(\bar{\phi}, \bar{\xi}, \bar{\eta}, \bar{g}, \bar{\varepsilon})$ is normal.

Hereafter, we assume $\varepsilon=1$, and drop it.

REMARK. A contact metric structure is normal if and only if the following tensor field vanishes :

$$
N(X, Y)=[\phi X, \phi Y]-\phi[\phi X, Y]-\phi[X, \phi Y]+\phi^{2}[X, Y]+2 d \eta(X, Y) \xi
$$

(cf. S. Sasaki [7], Theorem 11.1)

By the same method as in the case of Riemannian metric, we get the following, which we use later:

PROPOSITION 1. For an almost contact metric structure $(\phi, \xi, \eta, g)$ on $M$,

$$
\left(\nabla_{x} \phi\right) Y=\eta(Y) X-g(X, Y) \xi
$$

implies

(i) $\nabla_{x} \xi=\phi(X)$,

(ii) $\xi$ is a Killing vector field,

(iii) $d \eta(X, Y)=g(\phi X, Y)$.

Let $\left(M^{n}, g\right)$ be a pseudo-Riemannian manifold. Let $X$ and $Y$ be tangent vectors at a point of $M^{n}$. If $X$ and $Y$ satisfy

$$
g(X, X) g(Y, Y)-g(X, Y)^{2} \neq 0
$$

then we say that $X$ and $Y$ span a non-degenerate 2-plane $X \wedge Y$. This definition is independent of the choice of $X$ and $Y$ which span the 2-plane $X \wedge Y$. For a non-degenerate 2-plane $X \wedge Y$, we define a sectional curvature $K(X, Y)$ by

$$
K(X, Y)=\frac{g(R(X, Y) Y, X)}{g(X, X) g(Y, Y)-g(X, Y)^{2}}
$$

If $K(X, Y)$ is constant for all $X$ and $Y$ in $T_{x}\left(M^{n}\right)$ such that $X \wedge Y$ is a nondegenerate 2-plane, we call $\left(M^{n}, g\right)$ to be of constant curvature at $x$. If $\left(M^{n}, g\right)$ is of constant curvature at every point of $M^{n}, K(X, Y)$ is a function of $x \in M^{n}$, say $k(x)$. If $k(x)$ is constant on $M^{n}$, we call $\left(M^{n}, g\right)$ to be of constant curvature. It is known that if $\left(M^{n}, g\right)$ is of constant curvature at 
every point and if $n \geqq 3$, then $\left(M^{n}, g\right)$ is of constant curvature (J. A. Wolf [10], p. 57, Cor. 2.2.7). Suppose $\left(M^{n}, g\right)$ is of constant curvature $k$, then we have

$$
R(X, Y) Z=k\{g(Y, Z) X-g(X, Z) Y\}
$$

for all tangent vectors $X, Y$ and $Z$ (cf. J. A. Wolf [10], p. 56, Cor. 2.2.5).

Suppose we have a Sasakian manifold $M^{2 n+1}(\phi, \xi, \eta, g)$. Let

$$
D_{x}=\left\{X \in T_{x}\left(M^{2 n+1}\right) ; \eta(X)=0\right\} \text {. }
$$

For a non-null vector $X$ in $D_{x}, X$ and $\phi X$ span a non-degenerate 2-plane, and hence we can consider a sectional curvature $K(X)=K(X, \phi X)$. If $K(X)$ is constant for all non-null vectors $X$ in $D_{x}$, we call $\left(M^{2 n+1}, g\right)$ to be of constant $\phi$-sectional curvature at $x$. If $\left(M^{2 n+1}, g\right)$ is of constant $\phi$-sectional curvature at every point, $K(X)$ is a function of $x \in M^{2 n+1}$, say $k(x)$. In this case, if $k(x)$ is constant on $M^{2 n+1}$, we call $\left(M^{2 n+1}, g\right)$ to be of constant $\phi$-sectional curvature. If $\left(M^{2 n+1}, g\right)$ is of constant $\phi$-sectional curvature at every point and if $n \geqq 2,\left(M^{2 n+1}, g\right)$ is of constant $\phi$-sectional curvature (cf. $\mathrm{K}$. Ogiue [4]). Suppose $\left(M^{2 n+1}, g\right)$ is of constant $\phi$-sectional curvature $k$, then we have, for any tangent vectors $X, Y$ and $Z$,

$$
\begin{aligned}
4 R(X, Y) Z= & (k+3)\{g(Y, Z) X-g(X, Z) Y\}+(k-1)\{\eta(X) \eta(Z) Y \\
& -\eta(Y) \eta(Z) X+g(X, Z) \eta(Y) \xi-g(Y, Z) \eta(X) \xi \\
& +g(\phi Y, Z) \phi X+g(\phi Z, X) \phi Y-2 g(\phi X, Y) \phi Z\} .
\end{aligned}
$$

(cf. K. Ogiue [4]). Thus, if $\left(M^{2 n+1}, g\right)$ is of constant $\phi$-sectional curvature 1, it is of constant curvature 1 .

REMARK. If we do not assume $\varepsilon=1$, (3) should be

$$
\begin{aligned}
4 R(X, Y) Z= & (k+3 \varepsilon)\{g(Y, Z) X-g(X, Z) Y\} \\
& +(\varepsilon k-1)\{\eta(X) \eta(Z) Y-\eta(Y) \eta(Z) X\} \\
& +(k-\varepsilon)\{g(X, Z) \eta(Y) \xi-g(Y, Z) \eta(X) \xi \\
& +g(\phi Y, Z) \phi X+g(\phi Z, X) \phi Y-2 g(\phi X, Y) \phi Z\}
\end{aligned}
$$

2. Model spaces. Let $b_{s}^{n+1}$ be an "inner product" on $C^{n+1}$, defined by

$$
b_{s}^{n+1}(u, v)=\operatorname{Re}\left(-\sum_{i=1}^{s} u_{i} \bar{v}_{i}+\sum_{j=s+1}^{n+1} u_{j} \bar{v}_{j}\right) .
$$


Let $\widetilde{g}=g_{2 s}^{2 n+2}$ be a pseudo-Riemannian metric on $C^{n+1}$ defined by the parallel translation of $b_{s}^{n+1}$. Let $J$ be a complex structure on $C^{n+1}$ defined by the parallel translation of the map

$$
u \in C^{n+1} \longrightarrow \sqrt{-1} u .
$$

For $n \geqq 0$ and $0 \leqq s \leqq n$, let $M=S_{2 s}^{2 n+1}$ be a hypersurface of $C^{n+1}$ defined by

$$
S_{2 s}^{2 n+1}=\left\{u \in C^{n+1} ; b_{s}^{n+1}(u, u)=1\right\}
$$

and let $g=\widetilde{g} \mid S_{2 s}^{2 n+1}$. Then $(M, g)$ is a pseudo-Riemannian manifold of constant curvature 1, of dimension $2 n+1$ and of signature $2 s$ (cf. J. A. Wolf [10], pp. 62-68). If $s=0, M$ is nothing but the unit sphere $S^{2 n+1}$; S. Sasaki and Y. Hatakeyama [6] defined a Sasakian structure on it. Similarly, we can define a Sasakian structure on $M=S_{2 s}^{2 n+1}, n \geqq 0,0 \leqq s \leqq n$, as follows :

For $x \in M$, the tangent space of $M$ at $x$ is given by

$$
T_{x}(M)=\left\{X \in T_{x}\left(C^{n+1}\right) ; \widetilde{g}(X, x)=0\right\},
$$

where we consider $x$ as its position vector. Let $\xi$ be a vector field on $M$ defined by

$$
\xi: x \in M \longrightarrow \xi_{x}=J x,
$$

where $J x$ is considered as a tangent vector of $C^{n+1}$ at $x$ by the parallel translation. Since $J$ is skew-symmetric with respect to $\widetilde{g}, \widetilde{g}(J x, x)=0$; hence $J x$ is in $T_{x}(M)$, and

$$
g\left(\xi_{x}, \xi_{x}\right)=\widetilde{g}(x, x)=1 .
$$

Let $\eta$ be a 1 -form on $M$ defined by

$$
\eta(X)=g(\xi, X), \quad X \in \mathfrak{X}(M)
$$

Since $x \in M$ is a non-null vector in $C^{n+1}$, we have an orthogonal projection

$$
\pi: T_{x}\left(C^{n+1}\right) \longrightarrow T_{x}(M)
$$

with respect to $\widetilde{g}$, that is,

$$
\pi(X)=X-\widetilde{g}(x, X) x, \quad X \in T_{x}\left(C^{n+1}\right), \quad x \in M
$$


Let $\phi$ be a tensor field of type $(1,1)$ on $M$ defined by

$$
\phi=\pi \circ J
$$

It is easy to see that $(\phi, \xi, \eta, g)$ is an almost contact metric structure on $M$. We want to show that this structure is a Sasakian structure. According to Proposition 1 , it is sufficient to show

$$
\left(\nabla_{x} \phi\right) Y=\eta(Y) X-g(X, Y) \xi
$$

Consider $M$ to be a hypersurface of $C^{n+1}$. Then the vector field

$$
\zeta: x \in M \longrightarrow \zeta_{x}=x
$$

is a field of unit normal vectors to $M$ in $C^{n+1}$. For any vector fields $X$ and $Y$ tangent to $M$, we have the formulas of Gauss and Weingarten:

$$
\begin{aligned}
& D_{X} Y=\nabla_{X} Y+h(X, Y) \zeta, \\
& D_{X} \zeta=-A X
\end{aligned}
$$

where $D_{x}$ and $\nabla_{\boldsymbol{x}}$ denote covariant differentiations for $\widetilde{g}$ and $g$, respectively. $A$ is a field of symmetric endomorphisms (with respect to $g$ ) satisfying

$$
h(X, Y)=g(A X, Y)
$$

for tangent vectors $X$ and $Y$ (cf. L.P. Eisenhart [1]). Since the pseudoRiemannian metric $g$ is defined by the parallel translation,

$$
D_{x} \zeta=X
$$

for any tangent vector $X$ to $M$. Thus, (8), (9), (10) and (11) imply

$$
D_{X} Y=\nabla_{X} Y-g(X, Y) \zeta .
$$

Now, we have

$$
\left(\nabla_{x} \phi\right) Y=\nabla_{x}(\phi Y)-\phi \nabla_{x} Y
$$

for any vector fields $X$ and $Y$ tangent to $M$. We want to show that the right hand side of the above equation is nothing but the right hand side of (7). Using (12), we get 


$$
\begin{aligned}
\nabla_{X}(\phi Y) & =D_{x}(\phi Y)+g(X, \phi Y) \zeta \\
& =D_{X}(\pi J Y)+\widetilde{g}(X, \phi Y) \zeta .
\end{aligned}
$$

On the other hand, we have

$$
\begin{aligned}
D_{X}^{\prime}(\pi J Y) & =D_{X}(J Y-\widetilde{g}(\zeta, J Y) \zeta) \\
& =J D_{X} Y-\widetilde{g}(X, J Y) \zeta-\widetilde{g}\left(\zeta, J D_{X} Y\right) \zeta-\widetilde{g}(\zeta, J Y) X \\
\widetilde{g}(X, \phi Y) & -\widetilde{g}(X, J Y)=\widetilde{g}(X, \pi J Y-J Y) \\
& =\widetilde{g}(X,-\widetilde{g}(\zeta, J Y) \zeta) \\
& =0 .
\end{aligned}
$$

Thus (14) becomes

$$
\nabla_{x}(\phi Y)=J D_{X} Y-\widetilde{g}\left(\zeta, J D_{X} Y\right) \zeta-\widetilde{g}(\zeta, J Y) X .
$$

The second term of the right hand side of (13) is

$$
\begin{aligned}
\phi \nabla_{X} Y & =\pi J\left(D_{X} Y+g(X, Y) \zeta\right) \\
& =\pi J D_{X} Y+g(X, Y) \xi .
\end{aligned}
$$

Hence, (13), (15) and (16) imply

$$
\begin{aligned}
\left(\nabla_{x} \phi\right) Y & =J D_{X} Y-\widetilde{g}\left(\zeta, J D_{X} Y\right) \zeta-\widetilde{g}(\zeta, J Y) X-\pi J D_{x} Y-g(X, Y) \xi \\
& =\widetilde{g}\left(\zeta, J D_{x} Y\right) \zeta-\widetilde{g}\left(\zeta, J D_{X} Y\right) \zeta+g(\xi, Y) X-g(X, Y) \xi \\
& =\eta(Y) X-g(X, Y) \xi
\end{aligned}
$$

REMARK. If we replace (2),(3),(4) and (6) by

$$
\begin{aligned}
& H_{2 s-1}^{2 n+1}=\left\{u \in C^{n+1} ; b_{s}^{n+1}(u, u)=-1\right\}, \quad 1 \leqq s \leqq n+1, \\
& \bar{\xi}: x \in H_{2 s-1}^{2 n+1} \longrightarrow \bar{\xi}_{x}=-J x, \\
& \bar{\eta}(X)=-\bar{g}(\bar{\xi}, X), \quad \bar{g}=\widetilde{g} \mid H_{2 s-1}^{2 n+1}, \\
& \bar{\phi}=\bar{\pi} \circ J, \quad \bar{\pi} X=X+\widetilde{g}(x, X) x, \quad X \in T_{x}\left(H_{2 s-1}^{2 n+1}\right),
\end{aligned}
$$

then $H_{2 s-1}^{2 n+1}(\bar{\phi}, \bar{\xi}, \bar{\eta}, \bar{g},-1)$ is a Sasakian manifold and $H_{2 s-1}^{2 n+1}(\bar{\phi},-\bar{\xi},-\bar{\eta},-\bar{g},+1)$ 
is nothing but $S_{2(n-s+1)}^{2 n+1}(\phi, \xi, \eta, g)$ (cf. Example of $\S 1$ ).

It is known that $S_{2 s}^{2 n+1}$ is diffeomorphic to $R^{2 s} \times S^{2 n+1-2 s}$. Thus $S_{2 s}^{2 n+1}$ is simply connected for $s \neq n ; S_{2 n}^{2 n+1}$ is connected with infinite cyclic fundamental group. We define

$$
\begin{aligned}
& \widetilde{S_{2 s}^{2 n+1}}=S_{2 s}^{2 n+1} \quad \text { for } \quad s \neq n \\
& \widetilde{S_{2 n}^{2 n+1}}=\text { universal pseudo-Riemannian covering manifold of } S_{2 n}^{2 n+1}
\end{aligned}
$$

The Sasakian structure on $S_{2 n}^{2 n+1}$, which we defined above, induces a Sasakian structure on $\widetilde{S_{2 n}^{2 n+1}}$. We call $\widetilde{S_{2 s}^{2 n+1}}$ with the Sasakian structure to be the model spaces of Sasakian manifolds, and denote by $\widetilde{S}_{2 s}^{2 n+1}(\widetilde{\phi}, \tilde{\xi}, \widetilde{\eta}, \widetilde{g})$.

LemMa 1. Let $\left(M^{2 n+1}, h\right)$ be a pseudo-Riemannian manifold. Suppose $\left(M^{2 n+1}, h\right)$ is complete and of constant curvature $1, M^{2 n+1}$ is simply connected and $h$ is of signature $2 s, 0 \leqq s \leqq n, n \geqq 1$. Then, $\left(M^{2 n+1}, h\right)$ is isometric to the model space $\widetilde{S_{2 s}^{2 n+1}}$. (cf. J. A. Wolf [10], p. 68, Theorem 2.4.9).

Lemma 2. Suppose we have two Sasakian manifolds $M^{2 n+1}(\phi, \xi, \eta, g)$ and $\bar{M}^{2 n+1}(\bar{\phi}, \bar{\xi}, \bar{\eta}, \bar{g})$ such that $M$ and $\bar{M}$ are simply connected, $g$ and $\bar{g}$ have the same signature. If $(M, g)$ and $(\bar{M}, \bar{g})$ are complete and of constant curvature 1 , then there is an isometry

$$
f: M \longrightarrow \bar{M}
$$

such that $f_{*} \xi=\bar{\xi}, f^{*} \bar{\eta}=\eta, f_{*} \circ \phi=\bar{\phi} \circ f_{*} ;$ that is, $M(\phi, \xi, \eta, g)$ and $\bar{M}(\bar{\phi}, \bar{\xi}, \bar{\eta}, \bar{g})$ are equivalent.

ProOF. Let $x \in M$ and $\bar{x} \in \bar{M}$ be arbitrary points. Since $g$ and $\bar{g}$ have the same signature, we can find an isometry

$$
F: T_{x}(M) \longrightarrow T_{\bar{x}}(\bar{M})
$$

such that $F\left(\xi_{x}\right)=\bar{\xi}_{\bar{x}}, \bar{\eta}(F(X))=\eta(X)$ for $X \in T_{x}(M)$ and $F \circ \phi=\bar{\phi} \circ F$. Since $M$ and $\bar{M}$ are simply connected, and since $(M, g)$ and $(\bar{M}, \bar{g})$ are complete, we have a unique isometry

$$
f: M \longrightarrow \bar{M}
$$

such that $f(x)=\bar{x}$ and $f_{*} \mid T_{x}(M)=F$ (cf. J. A. Wolf [10], p. 61, Corollary 2.3.12). Since $f$ is an isometry and since $\xi$ is a Killing vector field by Proposition 1, 
$f_{*} \xi$ is a Killing vector field on $\bar{M}$. For any tangent vector $\bar{X}$ to $\bar{M}$, we have

$$
\bar{\nabla}_{\bar{x}}\left(f_{*} \xi\right)=f_{*}\left(\nabla_{f_{*} \cdot \bar{X}} \xi\right)=f_{*}\left(\phi f_{*}^{-1} \bar{X}\right)
$$

Hence, for $\bar{X} \in T_{\bar{x}}(\bar{M})$, we get

$$
\bar{\nabla}_{\bar{X}}\left(f_{*} \xi\right)=\bar{\phi} \bar{X}
$$

Thus, since $\bar{\xi}$ is a Killing vector field, (18), $\bar{\nabla}_{\bar{X}} \xi=\bar{\phi} \bar{X}$ and $\left(f_{*} \xi\right)_{\bar{x}}=\bar{\xi}_{\bar{x}}$ imply $f_{*} \xi=\bar{\xi}$, and hence $f^{*} \bar{\eta}=\eta$. Finally, for any $X \in \mathfrak{X}(M)$ and $\bar{Y} \in \mathfrak{X}(\bar{M})$, we have

$$
\begin{aligned}
\bar{g}\left(f_{*} \cdot \phi X, \bar{Y}\right) \cdot f & =\left(f^{*} \bar{g}\right)\left(\phi X, f_{*}^{-1} \bar{Y}\right)=g\left(\phi X, f_{*}^{-1} \bar{Y}\right) \\
& =d \eta\left(X, f_{*}^{-1} \bar{Y}\right)=\left(f^{*} d \bar{\eta}\right)\left(X, f_{*}^{-1} \bar{Y}\right) \\
& =d \bar{\eta}\left(f_{*} X, \bar{Y}\right) \cdot f=\bar{g}\left(\bar{\phi} \cdot f_{*} X, \bar{Y}\right) \cdot f,
\end{aligned}
$$

showing $f_{*} \circ \phi=\bar{\phi} \circ f_{*}$.

3. $D$-homothetic deformations. Suppose we have a Sasakian manifold $M^{2 n+1}(\phi, \xi, \eta, g)$. Let

$$
\bar{g}=\alpha g+\left(\alpha^{2}-\alpha\right) \eta \otimes \eta,
$$

where $\alpha$ is a non-zero constant, and let

$$
\bar{\xi}=(1 / \alpha) \xi, \quad \bar{\eta}=\alpha \eta, \quad \bar{\phi}=\phi
$$

Then $(\bar{\phi}, \bar{\xi}, \bar{\eta}, \bar{g})$ is a Sasakian structure on $M=M^{2 n+1}$, and we say that $M(\phi, \xi, \eta, g)$ is $D$-homothetic to $M(\bar{\phi}, \bar{\xi}, \bar{\eta}, \bar{g})$. If $(M, g)$ is of constant $\phi$-sectional curvature $k$, we have

$$
\begin{aligned}
\bar{K}(X) & =\bar{K}(X, \bar{\phi} X) \\
& =(1 / \alpha)\{k-3(\alpha-1)\}
\end{aligned}
$$

for any non-null vector $X \in D_{x}$, and hence $(M, \bar{g})$ is of constant $\phi$-sectional curvature $(1 / \alpha)\{k-3(\alpha-1)\}$. Thus if $k \neq-3$, and if we take $\alpha=(k+3) / 4$, $(M, \bar{g})$ is of constant $\phi$-sectional curvature 1 , and hence of constant curvature 1. (cf. S. Tanno [8], [9]). We summarize as follows :

Prorosition 2. A Sasakian manifold of constant $\phi$-sectional curvature $k \neq-3$ is D-homothetic to a Sasakian manifold of constant curvature 1. 
Let $M=M^{2 n+1}(\phi, \xi, \eta, g)$ be a Sasakian manifold.

Definition. We call a geodesic $x(t), \alpha<t<\beta$, to be $\xi$-geodesic (resp. D-geodesic) if $\phi(\dot{x}(t))=0($ resp. $\eta(\dot{x}(t))=0)$ for $\alpha<t<\beta$.

Definition. We call $M$ to be $\xi$-complete (resp. D-complete) if every $\xi$-geodesic (resp. $D$-geodesic) is complete.

Lemma 1. Let $M^{2 n+1}(\phi, \xi, \eta, g)$ be a Sasakian manifold. If $\left(M^{2 n+1}, g\right)$ is complete, then $\left(M^{2 n+1}, \vec{g}\right)$ is $\xi$ - and D-complete, where

$$
\vec{g}=\alpha g+\left(\alpha^{2}-\alpha\right) \eta \otimes \eta, \quad \alpha \neq 0 .
$$

PROOF. Let $\bar{\nabla}_{x}$ and $\nabla_{x}$ denote covariant differentiations for $\bar{g}$ and $g$, respectively. For any vector fields $X, Y$ and $Z$, we have

$$
\begin{aligned}
2 \vec{g}\left(\bar{\nabla}_{X} Y, Z\right)= & X \vec{g}(Y, Z)+Y \dot{g}(X, Z)-Z \vec{g}(X, Y) \\
& +\dot{g}([X, Y], Z)+\dot{g}([Z, X], Y)+\vec{g}([Z, Y], X) \\
= & 2 \alpha g\left(\nabla_{X} Y, Z\right)+\left(\alpha^{2}-\alpha\right)\{X(\eta(Y) \eta(Z))+Y(\eta(X) \eta(Z)) \\
& -Z(\eta(X) \eta(Y))+\eta([X, Y]) \eta(Z)+\eta([Z, X]) \eta(Y) ! \\
& +\eta([Z, Y]) \eta(X)\} .
\end{aligned}
$$

On the other hand, by the definition of contact metric structure,

$$
\begin{aligned}
2 g(\phi X, Y) & =2 d \eta(X, Y) \\
& =X \eta(Y)-Y \eta(X)-\eta([X, Y])
\end{aligned}
$$

Hence, we have

$$
\begin{aligned}
& \eta([X, Y])=X \eta(Y)-Y \eta(X)-2 g(\phi X, Y), \\
& \eta([Z, X])=Z \eta(X)-X \eta(Z)-2 g(\phi Z, X), \\
& \eta([Z, Y])=Z \eta(Y)-Y \eta(Z)-2 g(\phi Z, Y) .
\end{aligned}
$$

Thus we get

(3) $\bar{g}\left(\bar{\nabla}_{x} Y, Z\right)=\alpha g\left(\nabla_{X} Y, Z\right)+\left(\alpha^{2}-\alpha\right)\{(X \eta(Y)) \eta(Z)-g(\phi X, Y) \eta(Z)$

$$
-g(\phi Z, X) \eta(Y)-g(\phi Z, Y) \eta(X)\} \text {. }
$$


Now, suppose $x(t), \beta<t<\gamma$, be a geodesic in $M^{2 n+1}$ with respect to $\bar{g}$. Since $\bar{\xi}$ is a Killing vector field,

$$
\begin{aligned}
\bar{g}\left(\bar{\nabla}_{\dot{x}(t)} \bar{\xi}, \dot{x}(t)\right) & =(1 / 2)(L(\bar{\xi}) \bar{g})(\dot{x}(t), \dot{x}(t)) \\
& =0 .
\end{aligned}
$$

Hence we get

$$
\begin{aligned}
\dot{x}^{\prime}(t) \vec{\eta}(\dot{x}(t)) & =\dot{x}(t) \dot{g}\left(\bar{\xi}, \dot{x}^{\prime}(t)\right) \\
& =2 \dot{g}(\bar{\nabla} \dot{x}(t) \bar{\xi}, \dot{x}(t)) \\
& =0 .
\end{aligned}
$$

Since $\phi$ is skew symmetric with respect to $g$,

$$
\left.g\left(\phi \dot{x}^{\prime} t\right), \dot{x}^{\prime}(t)\right)=0 \text {. }
$$

If we put $X=Y=\dot{x}(t)$ in (3), then (4) and (5) imply

$$
\alpha g(\nabla \dot{x}(t) \dot{x}(t), Z)-\left(\alpha^{2}-\alpha\right) g(\phi Z, \dot{x}(t)) \eta(\dot{x}(t))=0 \text {. }
$$

This formula says that $x(t), \beta<t<\gamma$, is a geodesic with respect to $g$ if $x(t)$ is either $\xi$-geodesic or $D$-geodesic with respect to $\dot{g}$. Thus, since $\left(M^{2 n+1}, g\right)$ is complete, $\left(M^{2 n+1}, \bar{g}\right)$ is $\xi$ - and $D$-complete.

The following lemma is due to S. Tanno:

LEMMA 2. If a simply connected Sasnkian manifold $M=M^{2 n+1}(\phi, \xi, \eta, g)$ is $\xi$-and D-complete, and of constant curvature 1 , then it is complete.

ProOF. Let $\widetilde{S}$ be one of the model spaces such that the signature of $\widetilde{S}$ is the same as that of $M$. Let $\bar{x}(t), \alpha<t<\beta$, be a geodesic in $M$. We want to show that the geodesic can be extended for $\alpha<t<\beta+\varepsilon$ for some $\varepsilon>0$. We may suppose $0 \in(\alpha, \beta)$. Let us take any point $x_{0} \in \widetilde{S}$. Since $\widetilde{S}$ and $M$ are of constant curvature, we can find a local isomorphism $f_{0}$ such that $f_{0}\left(x_{0}\right)={ }^{-}(0)$. Let $X$ be a tangent vector to $\widetilde{S}$ at $x_{0}$ such that $f_{0 *}(X)=\dot{\bar{x}}(0)$, and let $x(t)$ be a geodesic in $\widetilde{S}$ such that $x(0)=x_{0}$ and $\dot{x}(0)=X$. Since $\widetilde{S}$ is complete, we can extend $x(t)$ for $-\infty<t<+\infty$. Thus we can extend the local isomorphism $f_{0}$ along $x(t)$ for $\alpha<t<\beta$, say $f_{1}$. To show that $\vec{x}(t)$ can be extended for $\alpha<t<\beta+\varepsilon$ for some $\varepsilon>0$, it is sufficient to show that $f_{0}$ can be extended along $x(t)$ for $\alpha<t \leqq \beta$. If $x(t)$ is either $\xi$-geodesic or 
$D$-geodesic it can be done, because $M$ is $\xi$ - and $D$-complete. So, we may suppose that $x(t)$ is neither $\xi$-geodesic nor $D$-geodesic. By considering a normal coordinate neighborhood of $\widetilde{S}$ at $x(\beta)$, we can find $t_{1} \in(0, \beta)$ such that, there exists $Y \in T_{x(t)}\left(\widetilde{S)}\right.$ such that $\widetilde{\eta}(Y)=0$ and the $D$-geodesic $y(t), y(0)=x\left(t_{1}\right)$ and $\dot{y}(0)=Y$, intersects the trajectory $L$ of $\tilde{\xi}$ passing through $x(\beta)$ at $z \in \widetilde{S \text {. }}$ Since $M$ is $D$-complete, we can extend $f_{1}$ along the $D$-geodesic $y(t)$, say $f_{2}$; especially, the domain of $f_{2}$ contains a neighborhood of $z$. Since $M$ is $\xi$-complete, we can extend $f_{2}$ along $L$, say $f_{3}$; in particular, the domain of $f_{3}$ contains a neighborhood of $x(\beta)$. Since $\widetilde{S}$ and $M$ are simply connected, these extensions are unique. Thus $f_{0}$ is extended along $x(t)$ for $\alpha<t \leqq \beta$.

\section{Main theorems.}

THEOREM 1. If a Sasakian manifold $M^{2 n+1}(\phi, \xi, \eta, g), n \geqq 1$, is complete, simply connected and of constant $\phi$-sectional curvature $k \neq-3$, then it is D-homothetic to the model space $\widetilde{S_{2 s}^{2 n+1}}$ of Sasakian manifolds, where

$$
\begin{aligned}
& 2 s=\text { the signature of } g \text { if } k>-3, \\
& 2 s=2 n-\text { the signature of } g \text { if } k<-3 .
\end{aligned}
$$

ProOF. Let

$$
\begin{aligned}
& \bar{g}=\alpha g+\left(\alpha^{2}-\alpha\right) \eta \otimes \eta, \\
& \bar{\xi}=(1 / \alpha) \xi, \quad \bar{\eta}=\alpha \eta, \quad \bar{\phi}=\phi, \\
& \alpha=(k+3) / 4 .
\end{aligned}
$$

Then Proposition 2 says that $M^{2 n+1}(\bar{\phi}, \bar{\xi}, \bar{\eta}, \bar{g})$ is a Sasakian manifold of constant curvature 1. According to Lemma 1 of $\S 3,\left(M^{2 n+1}, \vec{g}\right)$ is $\xi$ - and $D$-complete, and hence it is complete by Lemma 2 of $\S 3$. Since $\left(M^{2 n+1}, \tilde{g}\right)$ is complete, Lemma 1 of $\S 2$ says that it is isometric to $\widetilde{S}_{2 s}^{2 n+1}$, where

$$
\begin{aligned}
& 2 s=\text { the signature of } g \text { if } \alpha>0, \\
& 2 s=2 n-\text { the signature of } g \text { if } \alpha<0 .
\end{aligned}
$$

It is clear that $\alpha>0$ (resp. $\alpha<0$ ) is equivalent to $k>-3$ (resp. $k<-3$ ). Then, Lemma 2 of $\S 2$ says that $M^{2 n+1}(\bar{\phi}, \bar{\xi}, \bar{\eta}, \dot{g})$ is equivalent to the model space ${\widetilde{S_{2 s}}}_{2 n+1}$ of Sasakian manifold ; that is, the Sasakian manifold $M^{2 n+1}(\phi, \xi, \eta, g)$ is $D$-homothetic to $\widetilde{S_{2 s}^{2 n+1}}$.

COROllary. If a Sasakian manifold $M^{2 n+1}(\phi, \xi, \eta, g), n \geqq 1$, with a Riemannian metric $g$ is complete, simply connected and of constant 
$\phi$-sectional curvature $k \neq-3$, then it is D-homothetic to either the unit sphere $S^{2 n+1}$ if $k>-3$ or ${\widetilde{S_{2 n}^{2 n+1}}}^{2}$ if $k<-3$.

REMARK. The above Corollary was proved by S. Tanno [9] in the case of $k>-3$.

EXAMPLE. Let us consider the model space $\left(\widetilde{S}_{2 n}^{2 n+1}, \widetilde{g}\right) .{\widetilde{S_{2 n}^{2 n+1}}}_{\text {is }}$ the universal pseudo-Riemannian covering manifold of $S_{2 n}^{2 n+1}$, which is diffeomorphic to $R^{2 n} \times S^{1}$. Let us consider a $D$-homothetic deformation

$$
\vec{g}=-\widetilde{g}+2 \widetilde{\eta} \otimes \tilde{\eta},
$$

i.e., $\alpha=-1$ in (1) of $\S 3$. It is clear that $\bar{g}$ is a Riemannian metric of $\widetilde{S}_{2 n}^{2 n+1}$, and $(2)$ of $\S 3$ says that $\left(\widetilde{S}_{2 n}^{2 n+1}, \bar{g}\right)$ is of constant $\phi$-sectional curvature -7 .

THEOREM 2. Let $M_{i}=M_{i}^{2 n+1}\left(\phi_{i}, \xi_{i}, \eta_{i}, g_{i}\right), i=1,2, n \geqq 1$, be complete, simply connected Sasakian manifolds. Suppose they are of the same signature $2 s$ and of the same constant $\phi$-sectional curvature $k \neq-3$, then they are equivalent; that is, there is an isometry

$$
f: M_{1} \longrightarrow M_{2}
$$

such that $f_{*} \xi_{1}=\xi_{2}, f^{*} \eta_{2}=\eta_{1}$ and $f^{*} \circ \phi_{1}=\phi_{2} \circ f_{*}$.

ProOF. Theorem 1 says that $\bar{M}_{i}=M_{i}^{n+1}\left(\overline{\boldsymbol{\phi}}_{i}, \bar{\xi}_{i}, \bar{\eta}_{i}, \bar{g}_{i}\right), \quad i=1,2$, are equivalent to $\widetilde{S_{2 s}^{2 n+1}}$, where

$$
\begin{aligned}
\bar{g}_{i} & =\alpha g_{i}+\left(\alpha^{2}-\alpha\right) \eta_{i} \otimes \eta_{i}, \\
\bar{\xi}_{i} & =(1 / \alpha) \xi_{i}, \quad \bar{\eta}_{i}=\alpha \eta_{i}, \quad \bar{\phi}_{i}=\phi_{i}, \quad i=1,2, \\
\alpha & =(k+3) / 4 .
\end{aligned}
$$

Hence, Lemma 2 of $\S 2$ implies that $\bar{M}_{1}$ and $\bar{M}_{2}$ are equivalent; that is, there is an isometry

$$
f: \bar{M}_{1} \longrightarrow \bar{M}_{2}
$$

such that $f_{*} \bar{\xi}_{1}=\bar{\xi}_{2}, f^{*} \bar{\eta}_{2}=\bar{\eta}_{1}$ and $f_{*} \circ \bar{\phi}_{1}=\bar{\phi}_{2} \circ f_{*}$. Since

$$
g_{i}=(1 / \alpha) \bar{g}_{i}+\left(\left(1 / \alpha^{2}\right)-(1 / \alpha)\right) \overline{\boldsymbol{\eta}}_{i} \otimes \overline{\boldsymbol{\eta}}_{i}, \quad i=1,2,
$$

$f$ is an isometry 


$$
f: M_{1} \longrightarrow M_{2} \text {. }
$$

Moreover, we have

$$
\begin{aligned}
& f_{*} \xi_{1}=f_{*}\left(\alpha \overline{\xi_{1}}\right)=\alpha \bar{\xi}_{2}=\xi_{2}, \\
& f^{*} \eta_{2}=f^{*}\left((1 / \alpha) \bar{\eta}_{2}\right)=(1 / \alpha) \bar{\eta}_{1}=\eta_{1}, \\
& f_{*} \circ \phi_{1}=f_{*} \circ \bar{\phi}_{1}=\bar{\phi}_{2} \circ f_{*}=\phi_{2} \circ f_{*} .
\end{aligned}
$$

Thus $f$ gives the equivalence of $M_{1}$ and $M_{2}$.

5. Sasakian manifold with $\boldsymbol{R}(\boldsymbol{X}, \boldsymbol{Y}) \cdot \boldsymbol{R}=\mathbf{0}$. Let $M^{2 n+1}(\phi, \xi, \eta, g)$ be a Sasakian manifold. Then, by the definition of Sasakian manifold, we get

$$
\begin{aligned}
R(X, \xi) Y & =\nabla_{X} \nabla_{Y} \xi-\nabla_{\nabla_{X}} \xi \quad(\because \xi \text { is a Killing vector field }) \\
& =\nabla_{X}(\phi Y)-\phi\left(\nabla_{X} Y\right) \\
& =\left(\nabla_{X} \phi\right) Y+\phi\left(\nabla_{X} Y\right)-\phi\left(\nabla_{X} Y\right) \\
& =\eta(Y) X-g(X, Y) \xi \\
R(X, Y) \xi & =\nabla_{X} \nabla_{Y} \xi-\nabla_{Y} \nabla_{X} \xi-\nabla_{[X, Y]} \xi \\
& =\nabla_{X}(\phi Y)-\nabla_{Y}(\phi X)-\phi([X, Y]) \\
& =\left(\nabla_{X} \phi\right) Y+\phi\left(\nabla_{X} Y\right)-\left(\nabla_{Y} \phi\right) X-\phi\left(\nabla_{Y} X\right)-\phi([X, Y]) \\
& =\eta(Y) X-g(X, Y) \xi-(\eta(X) Y-g(Y, X) \xi) \\
& =\eta(Y) X-\eta(X) Y
\end{aligned}
$$

for any vector fields $X$ and $Y$. Suppose $R(X, Y) \cdot R=0$ for all tangent vectors $X$ and $Y$, where $R(X, Y)$ operates on $R$ as a derivation of the tensor algebra at each point. Now, let $X$ and $Y$ be tangent vectors such that $\eta(X)=\eta(Y)=0$ and $g(X, Y)=0$. Then, using (1) and (2) above,

$$
\begin{aligned}
( & R(X, \xi) \cdot R)(X, Y) Y \\
= & R(X, \xi) R(X, Y) Y-R(R(X, \xi) X, Y) Y-R(X, R(X, \xi) Y) Y-R(X, Y) R(X, \xi) Y \\
= & \eta(R(X, Y) Y) X-g(X, R(X, Y) Y) \xi-R(\eta(X) X-g(X, X) \xi, Y) Y \\
& -R(X, \eta(Y) X-g(X, Y) \xi) Y-R(X, Y)(\eta(Y) X-g(X, Y) \xi) \\
= & \eta(R(X, Y) Y) X-g(X, R(X, Y) Y) \xi+g(X, X) R(\xi, Y) Y
\end{aligned}
$$




$$
=\eta(R(X, Y) Y) X-g(X, R(X, Y) Y) \xi-g(X, X) \eta(Y) Y+g(X, X) g(Y, Y) \xi
$$

Hence,

$$
\eta(R(X, Y) Y) X-g(X, R(X, Y) Y) \xi+g(X, X) g(Y, Y) \xi=0
$$

Thus, considering $\xi$-component of (3), we get

$$
g(X, R(X, Y) Y)=g(X, X) g(Y, Y),
$$

showing that $\left(M^{2 n+1}, g\right)$ is of constant $\phi$-sectional curvature 1 , and hence it is of constant curvature 1 .

THEOREM 3. A Sasakian manifold satisfying $R(X, Y) \cdot R=0$ for all tangent vectors $X$ and $Y$ is of constant curvature 1 .

6. Sasakian manifold $M^{2 n+1}$ which is isometrically immersed in $\boldsymbol{E}_{s}^{2 n+2}$. Let $E_{s}^{n}$ be a Euclidean space $R^{n}$ with a pseudo-Riemannian metric $\widetilde{g}_{s}$ which is defined by the parallel displacement of the "inner product"

$$
<x, y>=-\sum_{i=1}^{s} x^{i} y^{i}+\sum_{j=s+1}^{n} x^{j} y^{j}
$$

Then the signature of $\widetilde{g}_{s}$ is $s$, and $E_{s}^{n}$ is complete and of constant curvature 0 (cf. J. A. Wolf [10], \$2.4).

Let $M^{2 n+1}(\phi, \xi, \eta, g)$ be a Sasakian manifold. Suppose we have an isometric immersion

$$
f: M^{2 n+1} \longrightarrow E_{s}^{2 n+2} .
$$

For each $x \in M^{2 n+1}$, we can choose a unit vector field $\zeta$ normal to $M^{2 n+1}$ on some neighborhood $U$ of $x$ :

$$
\widetilde{g_{s}}(\zeta, \zeta)=\varepsilon, \quad \varepsilon=1 \quad \text { or }-1 \quad \text { on } U
$$

For any vector fields $X$ and $Y$ on $U$ tangent to $M^{2 n+1}$, we have the formulas of Gauss and Weingarten:

$$
\begin{aligned}
& D_{X} Y=\nabla_{X} Y+\varepsilon h(X, Y) \zeta, \\
& D_{X} \zeta=-A X,
\end{aligned}
$$


where $D_{X}$ and $\nabla_{x}$ denote covariant differentiations for $\tilde{y}_{s}$ and $g$, respectively. $A$ is a field of symmetric endomorphisms which corresponds to the second fundamental form $h$, that is, $h(X, Y)=g(A X, Y)$ for all tangent vectors $X$ and $Y$. The equation of Gauss expresses the curvature tensor $R$ of $M^{2 n+1}$ by means of $A$ :

$$
R(X, Y) Z=\varepsilon\{g(Z, A Y) A X-g(Z, A X) A Y\}
$$

This equation implies

$$
R(X, \xi) Y=\varepsilon\{\eta(A Y) A X-g(A X, Y) A \xi\}
$$

On the other hand, we have (1) of $\S 5$ :

$$
R(X, \xi) Y=\eta(Y) X-g(X, Y) \xi
$$

Suppose the isometric immersion $f: M^{2 n+1} \longrightarrow E_{s}^{2 n+2}$ is proper, that is, $A$ can be expressed by a real diagonal matrix with respect to a certain orthonormal frame at each point of $M^{2 n+1}$ (cf. A. Fialkow [2], p.764). Let $\left\{e_{1}, e_{2}, \cdots, e_{2 n+1}\right\}$ be an orthonormal basis of $T_{x_{0}}\left(M^{2 n+1}\right)$ such that $A$ is expressed by a diagonal matrix with respect to $\left\{e_{1}, e_{2}, \cdots e_{2 n+1}\right\}$, i.e.,

$$
A e_{i}=\rho_{i} e_{i}, \quad 1 \leqq i \leqq 2 n+1, \quad \rho_{i} \in R
$$

(2), (3) with $X=e_{i}, \quad Y=e_{j}$ and (4) imply

$$
\eta\left(e_{j}\right) e_{i}-g\left(e_{i}, e_{j}\right) \xi=\varepsilon\left\{\rho_{i} \rho_{j} \eta\left(e_{j}\right) e_{i}-\rho_{i} g\left(e_{i}, e_{j}\right) A \xi\right\}
$$

If $i \neq j$, (5) implies

$$
\eta\left(e_{j}\right) e_{i}=\varepsilon \rho_{i} \rho_{j} \eta\left(e_{j}\right) e_{i}
$$

Hence $\varepsilon \rho_{i} \rho_{j}=1$ for all $i \neq j$, or $\eta\left(e_{j}\right)=0$ for some $j$.

(a) Suppose $\varepsilon \rho_{i} \rho_{j}=1$ for all $i \neq j$. Then $\rho_{i} \neq 0$ for all $i$, and $\rho_{1}=\rho_{2}$ $=\cdots=\rho_{2 n+1}=\rho$. Thus $\varepsilon \rho^{2}=1$. This implies $\varepsilon=1$ and $\rho^{2}=1$.

(b) Suppose $\eta\left(e_{j_{0}}\right)=0$ for some $j_{0}$. Then (5) implies

$$
\xi=\varepsilon \rho_{j_{0}} A \xi
$$

Hence $\rho_{j_{0}} \neq 0$ and $A \xi=\left(1 / \varepsilon \rho_{j_{0}}\right) \xi$, i. e, $\xi$ is an eigenvector of $A$ with eigenvalue 
$1 / \varepsilon \rho_{j_{0}}$. We may suppose $e_{1}=\xi$, and hence $\eta\left(e_{i}\right)=0$ for $2 \leqq i \leqq 2 n+1$. implies

$$
K\left(e_{i}, \xi\right)=\varepsilon \rho_{1} \rho_{i}
$$

(3) implies

$$
K\left(e_{i}, \xi\right)=1
$$

for $2 \leqq i \leqq 2 n+1$. Hence we get $\rho_{1} \rho_{i}=\varepsilon$ for $2 \leqq i \leqq 2 n+1$, and hence $\rho_{2}=\rho_{3}$ $=\cdots=\rho_{2 n+1}=\rho$. Consequently, $A X=\rho X$ for any tangent vector $X$ such that $\eta(X)=0$. Thus (1) implies $\left(M^{2 n+1}, g\right)$ is of constant $\phi$-sectional curvature $\varepsilon \rho^{2}$, hence we have (3) of $\S 1$ with $k=\varepsilon \rho^{2}$. Now, if we assume $n \geqq 2$, we can find non-null tangent vectors $X$ and $Y$ such that $\eta(X)=\eta(Y)=0, g(X, Y)=0$ and $g(\phi X, Y)=0$. Then (3) of $\$ 1$ and (1) of this section give

$$
4 R(X, Y) X=-(k+3) g(X, X) Y
$$

and

$$
R(X, Y) X=-\varepsilon \rho^{2} g(X, X) Y
$$

respectively. Hence we get

$$
\frac{k+3}{4}=\varepsilon \rho^{2}
$$

Since $k=\varepsilon \rho^{2}$, this equation implies $\varepsilon \rho^{2}=1$, that is, $\rho^{2}=\varepsilon$. Hence $\varepsilon=1$ and $\rho^{2}=1$. Since $\rho_{1} \rho=\varepsilon$, we get $\rho_{1}=\rho$.

Summarizing (a) and (b), if $n \geqq 2$, we have $\varepsilon=1, A=\rho$ and $\rho^{2}=1$. We may suppose $\rho=1$, since the change $\zeta \longrightarrow-\zeta$ implies $A \longrightarrow-A, \rho=(1 /(2 n+1))$. $\operatorname{Tr} A$ is a differentiable function on $U$.

Now, let us suppose $n \geqq 2$. Consider the $R^{2 n+2}$-valued function

$$
x \in U \subset M^{2 n+1} \longrightarrow \zeta_{x}+f(x) \in R^{2 n+2} .
$$

For any tangent vector $X$ to $M^{2 n+1}$, we have

$$
\begin{aligned}
D_{f_{*} x}(\zeta+f) & =f_{*}(-A X+X) \\
& =0
\end{aligned}
$$

This implies that $\xi+f$ is a constant map $M^{2 n+1} \longrightarrow \alpha \in R^{2 n+2}$, and hence 


$$
\begin{aligned}
<f(x)-\alpha, f(x)-\alpha> & =\left\langle\zeta_{x}, \zeta_{x}>\right. \\
& =1
\end{aligned}
$$

for $x \in U$. Thus $f(U)$ lies on the hypersurface $S_{s}^{2 n+1}(\alpha)$, which is the hypersurface $S_{s}^{2 n+1}$ translated by the parallel translation $\beta \longrightarrow \alpha+\beta, \beta \in R^{2 n+2}$. Let $M^{\prime}=\left\{x \in M^{2 n+1}: f(x) \in S_{s}^{2 n+1}(\alpha)\right\}$. Then the above argument says that $M^{\prime}$ is open. Similarly, $M^{2 n+1}-M^{\prime}$ is open, showing $M^{\prime}$ to be closed. Thus, since $M^{2 n+1}$ is connected, $M^{\prime}=M^{2 n+1}$, i.e., $f\left(M^{2 n+1}\right)$ lies on $S_{s}^{2 n+1}(\alpha)$. In particular, $\left(M^{2 n+1}, g\right)$ is of constant curvature 1 .

THEOREM 4. Suppose we have a complete Sasakian manifold $M^{2 n+1}(\phi, \xi, \eta, g), n \geqq 2$, which is properly and isometrically immersed in $E_{s}^{2 n+2}$. Then

(i) if $0 \leqq s \leqq 2 n-1$, then $s$ is even, the immersion is an isometric imbedding and $M^{2 n+1}(\phi, . \xi, \eta, g)$ is equivalent to $\widetilde{S_{s}^{2 n+1}}$,

(ii) if $2 n \leqq s \leqq 2 n+2$, then $s=2 n$ and $M^{2 n+1}(\phi, \xi, \eta, g)$ is a pseudoRiemannian covering manifold of $S_{2 n}^{2 n+1}$ and the immersion induces the covering projection, naturally.

We need the following Lemma :

LEMMA. Let $M_{1}=\left(M_{1}^{n}, h_{1}\right)$ and $M_{2}=\left(M_{2}^{n}, h_{2}\right)$ be pseudo-Riemannian manifolds with the same dimension and signature. Suppose $M_{1}$ and $M_{2}$ are of the same constant curvature $k$, and suppose we have an isometric immersion

$$
f: M_{1} \longrightarrow M_{2}
$$

Then, if $M_{1}$ is complete, $M_{2}$ is also complete and the isometric immersion $f$ is a covering projection (cf. S. Kobayashi-K. Nomizu [3], Theorem 4.6).

PROOF. Let $y_{2}$ be an arbitrary point of $M_{2}$. Let us take $x_{1} \in M_{1}$ and let $x_{2}=f\left(x_{1}\right)$. Then we can join $x_{2}$ and $y_{2}$ by a broken geodesic $L_{2}$. Since $M_{1}$ is complete, there is a broken geodesic $L_{1}$ in $M_{1}$ such that $f\left(L_{1}\right)=L_{2}$, showing that $f$ is an onto mapping.

Let $x_{2}(t), \alpha<t<\beta$, be a geodesic in $M_{2}$. Then, since $M_{1}$ is complete, we have a geodesic $x_{1}(t),-\infty<t<+\infty$, such that $f\left(x_{1}(t)\right)=x_{2}(t)$ for $\alpha<t<\beta$. Since $f$ is an isometric immersion, there is a neighborhood $U$ of $x_{1}(\alpha)$ (resp. $x_{1}(\beta)$ ) such that $f \mid U$ is an isometry of $U$ onto $f(U)$ which is a 
neighborhood of $f\left(x_{1}(\alpha)\right)$ (resp. $f\left(x_{1}(\beta)\right)$. Thus the geodesic $x_{2}(t), \alpha<t<\beta$, can be extended for $\alpha-\varepsilon^{\prime}<t<\beta+\varepsilon^{\prime \prime}$ for some positive constants $\varepsilon^{\prime}$ and $\varepsilon^{\prime \prime}$, showing $M_{2}$ to be complete.

Let us consider the universal pseudo-Riemannian covering manifolds $\widetilde{M}_{1}$ and $\widetilde{M}_{2}$ of $M_{1}$ and $M_{2}$ with projections $p_{1}$ and $p_{2}$, respectively. Let $x_{1}$ be an arbitrary point of $M_{1}$, choose $y_{1} \in p_{1}^{-1}\left(x_{1}\right)$ and $y_{2} \in p_{2}^{-1}\left(f\left(x_{1}\right)\right)$. Let $V_{y_{1}}, U_{x_{1}}$, $U_{f\left(x_{1}\right)}$ and $V_{y_{2}}$ be neighborhoods of $y_{1}, x_{1}, f\left(x_{1}\right)$ and $y_{2}$, respectively, such that $p_{1}, f$ and $p_{2}$ are isometries of $V_{y_{1}}, U_{x_{1}}$ and $V_{y_{2}}$ onto $U_{x_{1}}, U_{f\left(x_{1}\right)}$ and $U_{f\left(x_{1}\right)}$, respectively. Then we have an isometry

$$
F=: p_{2}^{-1} f p_{1}: V_{y_{1}} \longrightarrow V_{y_{2}}
$$

Since $\bar{M}_{1}$ and $\widetilde{M}_{2}$ are complete, simply connected and of constant curvature $k$, the local isometry $F$ has a unique extension, say $F$; that is, an isometry $F: \widetilde{M}_{1} \longrightarrow \widetilde{M}_{2}$. Since this extension can be done along all (broken) geodesics passing through $y_{1}$, we have

$$
p_{2} \circ F=f \circ p_{1}
$$

which shows that $f$ is a covering projection, since $f$ is a continuous and open mapping.

ProOF OF THEOREM 4. The above Lemma says that the isometric immersion is a covering projection $M^{2 n+1} \longrightarrow S_{s}^{2 n+1}(\alpha)$. If $0 \leqq s \leqq 2 n-1, s$ is even, then $S_{s}^{2 n+1}(\alpha)$ is simply connected, hence the covering projection is an isometry. Thus the Theorem follows from Lemma 2 of $\$ 2$.

\section{REFERENCES}

[ 1] L. P. Eisenhart, Riemannian Geometry, Chapter IV, Princeton University Press, 1949.

[2] A. Fialkow, Hypersurfaces of a space of constant curvature, Ann. of Math., 39(1938), 762-785.

[3] S. Kobayashi AND K. Nomizu, Foundations of differential geometry, Volume 1, Interscience, 1963.

[4] K. OGIUE, On almost contact manifolds admitting axiom of planes or axiom of free mobility, Kodai Math. Sem. Rep., 16(1964), 223-232.

[5] S. SASAKI, On differentiable manifolds with certain structures which are closely related to almost contact structure I, Tôhoku Math. J., 12(1960), 459-476.

[6] S. SASAKI AND Y. HATAKEYAMA, On differentiable manifolds with contact metric structures, J. of Math. Soc. of Japan, 14(1962), 249-271.

[ 7 ] S. SASAKI, Almost contact manifolds, Part I, Lecture note, Math. Inst, of Tôhoku Univ., 1965. 
[ 8 ] S. TANNO, Partially conformal transformations with respect to $(m-1)$-dimensional distributions of $m$-dimensional Riemannian manifolds, Tôhoku Math. J., 17(1965), 358-409.

[9] S. TANNO, The topology of contact Riemannian manifolds, Illinois J. of Math., 12(1968), 700-717.

[10] J.A. Wolf, Spaces of constant curvature, McGraw-Hill Book Co., 1967.

MATHEMATICAL INSTITUTE

TÔHOKU UNIVERSITY

SENDAI, JAPAN 